One of the primary concerns following endovascular aneurysm treatment is recurrence, and close imaging followup is often required. High resolution MRI is being employed in an increasingly wide variety of pathologies, but its use as a surveillance tool following flow diversion has not been extensively explored. We present three cases where high resolution MRI was performed following flow diversion for intracranial aneurysms and discuss its utility in this patient population.

\begin{tabular}{|c|c|c|c|c|c|}
\hline \multicolumn{6}{|c|}{ MRI sequence parameters } \\
\hline MRI sequence & $\begin{array}{l}\mathrm{TR} / \mathrm{TE} \\
\text { (ms) }\end{array}$ & $\begin{array}{l}\text { FOV } \\
\left(\mathrm{mm}^{2}\right)\end{array}$ & Matrix & $\begin{array}{l}\text { Thickness } \\
\text { (mm) }\end{array}$ & $\begin{array}{l}\text { Scan time } \\
\text { (min:sec) }\end{array}$ \\
\hline 3D TOF & $22 / 3.6$ & $200 \times 181$ & $384 \times 331$ & 0.5 & 6:50 \\
\hline $\begin{array}{l}\text { 3D DANTE T1w BB } \\
\text { SPACE }\end{array}$ & $800 / 21$ & $162 \times 162$ & $196 \times 192$ & 0.8 & $5: 47$ \\
\hline 3D T2w BB SPACE & $\begin{array}{l}1000 / \\
118\end{array}$ & $200 \times 200$ & $384 \times 380$ & 0.5 & $4: 18$ \\
\hline
\end{tabular}

Disclosures J. Guan: None. S. McNally: None. A. de Havenon: None. P. Tuassky: 2; C; Covidien. S. Kim: None. M. Park: None.

\section{E-074 AN ANALYSIS TO FINAL DIAGNOSIS IN CEREBROVASCULAR ACCIDENT (CVA) PROTOCOL ACTIVATION PATIENTS: SINGLE CENTER STUDY}

B Moon, S Park, K Jang, D Jang. Neurosurgery, Incheon St. Mary's Hospital, Incheon, Republic of Korea

\subsection{6/neurintsurg-2016-012589.146}

Introduction The cerebrovascular accident(CVA) is a disease that can result in life threatening or permanent neurologic sequelae. Although CVA is a world-wide problem, the burden of CVA is particularly serious in Asia; its mortality is higher than in Europe or North America. For patients with CVA occurrence for quick diagnosis and treatment of the CVA protocol, we have many hospitals are trying to maintain the life of patients without neurological sequelae and rapid treatment. The classification of patients is made to only initial patient questionnaire in this protocol. The cases that are often mistaken for CVA have occurred. This study was conducted for determine the method for more accurate patients classification and then to reduce the misdiagnosis.

Methods The authors conducted a retrospective analysis of CVA protocol activation patient data that were collected at Incheon St. Mary's hospitals over a 8 year period (from January 2008 to July 2015).

The authors were reviewed for CVA protocol activation patients with early symptoms, radiologic image study, lab finding, electronic medical record(EMR) and including the final diagnosis for the patient through the EMR. The diagnosis was classified as a acute CVA such as cerebral infarction, transient ischemic attack (TIA), intracerebral hemorrhage (ICH), intraventricular hemorrhage (IVH), subarachnoid hemorrhage(SAH), arteriovenous malformation (AVM) and diseases that can be confused with this disease such as brain tumor, seizure, traumatic intracranial hemorrhage, medical problem (i.g hyponatremia, pneumonia, myocardial infarction, hypoglycemia), drug abuse, peripheral neuropathy, spinal problem, neuromuscular disease, psychiatric problem.
Results In total, 2191 patients met the inclusion criteria of this study. These patients were diagnosed with cerebral infarction (1187, 54.17\%), TIA (241, 10.99\%), ICH (376, 17.16\%), SAH (134, 6.11\%), AVM (10, 0.45\%), IVH (4, 0.18\%), brain tumor $(22,1.00 \%)$, traumatic hemorrhage $(75,3.42 \%)$, medical problem $(31,1.41 \%)$, seizure $(22,1.00 \%)$, drug abuse $(6,0.27 \%)$, psychiatric disorder $(4,0.18 \%)$, peripheral neuropathy $(17,0.77 \%)$, neuromuscular disease $(5,0.22 \%)$, etc $(57,2.60 \%)$.

Conclusions This analysis showed that the $89 \%$ of patients in the CVA protocol activation were the acute CVA disease. But, the $11 \%$ of patients in the CVA protocol activation were the others disease. Well-trained doctors should be conducted initial history taking and accurate neurological examination for reduce to misdiagnosis. And then, we could achieve a cost effective rapid treatment.

\section{CVA protocol}

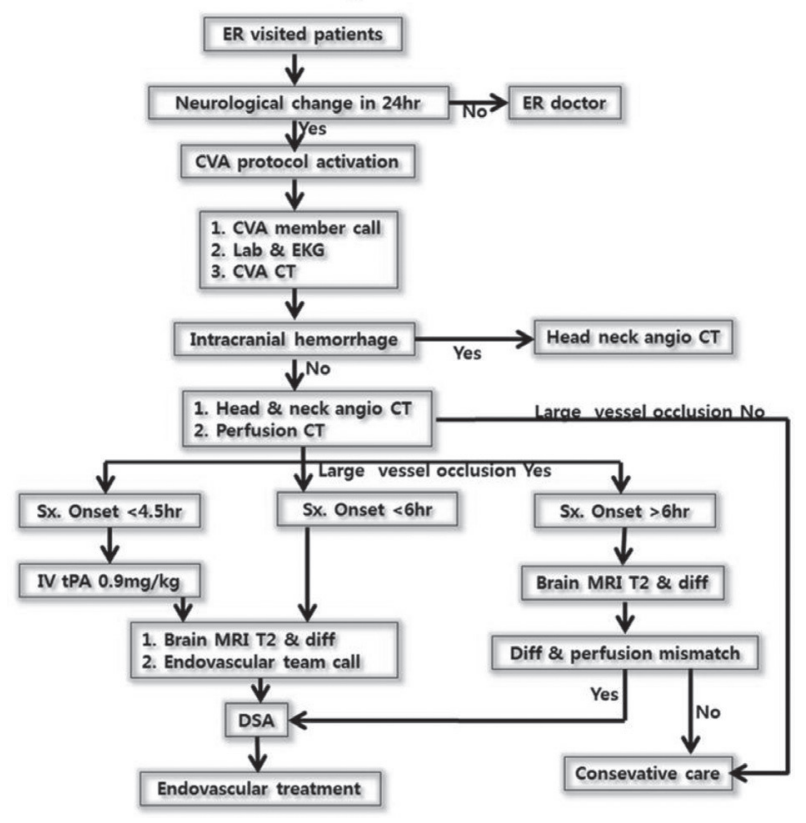

Abstract E-074 Figure 1

Disclosures B. Moon: None. S. Park: None. K. Jang: None. D. Jang: None.

\section{E-075 PERCUTANEOUS THORACOLUMBAR DECOMPRESSION COMBINED WITH PERCUTANEOUS PEDICLE SCREW FIXATION AND FUSION: A METHOD FOR TREATING SPINAL DEGENERATIVE PAIN IN A BIPLANE ANGIOGRAPHY SUITE WITH THE AVOIDANCE OF GENERAL ANESTHESIA}

B Chopko. Neurosurgery, Stanford University, Henderson, NV

\subsection{6/neurintsurg-2016-012589.147}

Objective Spondylytic degeneration of the axial lumbar spine is a major cause of pain and disability. Recent advances in spinal surgical instrumentation, including percutaneous access and fusion techniques, have made possible the performance of instrumented fusion through small incisions. By blending 